\title{
Eclipsing Binaries: Precise Clocks to Detect Extrasolar Planets
}

\author{
Emil Kundra, Theodor Pribulla, Martin Vaňko \\ and Ľubomír Hambálek $\dagger$
}

Astronomical Institute of the Slovak Academy of Sciences 05960 Tatranská Lomnica, Slovakia, email: kundra@ta3.sk

\begin{abstract}
Project Dwarf is a new observing campaign focused on the detection of substellar companions to low-mass (composed of late-type, subdwarf (sd) or/and white dwarf (WD) components) detached eclipsing binaries using minima timing. The crucial condition for the object selection for this campaign is possibility to determine times of the minima with high precision. This is naturally fullfilled for eclipsing binaries with deep and narrow minima or systems hosting a WD component showing fast ingress or egress.

The observing project includes three groups of close eclipsing binaries indicating presence of substellar circum-binary components:

(i) systems with $\mathrm{K}$ or/and $\mathrm{M}$ dwarf components

(ii) systems with hot subdwarf (sd) and $\mathrm{M}$ dwarf components

(iii) systems with white dwarf (WD) component(s).

The sample of the eclipsing systems have orbital periods in range of 0.1 to almost 3 days and their brightness fits possibilities of small telescopes equipped with a low-end CCD camera and at least VRI filter set. Such kind of telescopes allow us to develop observing network including also amateur astronomers.
\end{abstract}

Keywords. stars: planetary systems, binaries: eclipsing, techniques: photometric

\section{Introduction to project DWARF}

With the continuing discovery of extrasolar planets planetary formation in binary systems has become an important issue (Lee et al. 2009). In general, we can consider planetary companions to binary stars in two types of hierarchical planet-binary configurations: first S-type planets which orbit just one of the stars, with the binary period being much longer than that of the planet; second, P- type or circumbinary planets, where the planet simultaneously orbits both stars, and the planetary orbital period is much longer than that of the binary (Dvorak 1984, Muterspaugh et al. 2007). Simulations show either of above possibilities has a large range of stable configurations (see e.g., Broucke 2001). Recent theoretical studies (e.g., Pierens \& Nelson 2008) have predicted that P-type planets can form and survive over long timescales. Characterization of such planets is potentially of great interest because they can lead to a better understanding of the formation and evolution of planetary systems around close binary stars, which can be rather different from the case of single stars (Lee et al. 2009).

Hereafter we will consider the P-type or circumbinary planets only. The detection of circumbinary planets is far from being easy. Three principal techniques are:

$\dagger$ This article was supported by the VEGA Grant No.2/0094/11 and by the realization of the Project ITMS No. 26220120029, based on the supporting operational Research and development program financed from the European Regional Development Fund. 

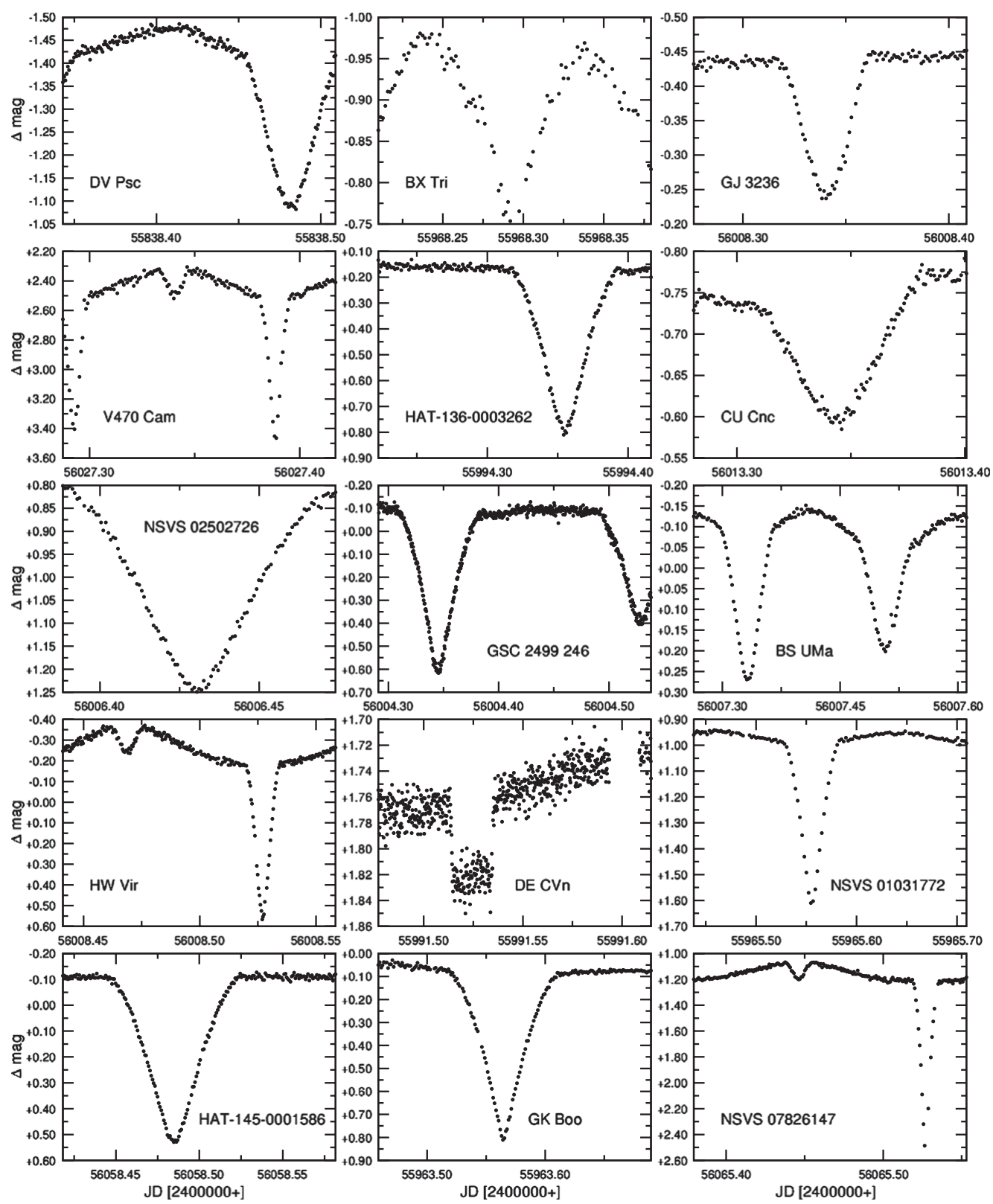

Figure 1. A sample of LCs obtained at the Stará Lesná observatory using either $0.5 \mathrm{~m}$ Newtonian or $0.6 \mathrm{~m}$ Cassegrain telescopes. LCs of most objects were obtained in the $I$ passband (except V470 Cam, HW Vir, DE CVn and NSVS 07826147). The Julian dates are geocentric.

(i) precise radial-velocity (RV) measurements to detect the wobble of the binary mass center (Konacki et al. 2009),

(ii) photometric detection of transits of the planet(s) across the disks of the components of the inner binary (Doyle et al. 2011), and

(iii) timing of the inner binary eclipses (Lee et al. 2009). 
The classical RV technique is complicated by the fact that the large RV changes of the binary components mask the small wobble resulting from the circumbinary planet(s). In the case of close binaries, the situation is exacerbated by the tidal spin-up of the components, where the projected rotational velocity ( $\mathrm{v} \sin \mathrm{i}$ ) often exceeds $100 \mathrm{~km} \mathrm{~s}^{1}$ (see the DDO close-binary project, Pribulla et al., 2009 and references therein). This makes the RV precision insufficient to detect any systemic velocity changes. In fact, there are hardly any binaries where the systemic-velocity changes revealed a third component (unseen in spectra).

The second technique - to detect circumbinary planets searching for transits of a substellar companion across a close binary - requires very long photometric runs with excellent accuracy. Assuming that the orbital planes of the underlying binary and the outer orbit of the substellar body are close to being coplanar, the method should be advantageous for edge-on eclipsing binaries (EBs). Three such systems were found in the Kepler satellite photometry: Kepler-16b (Doyle et al., 2011), Kepler-34b and Kepler-35b (Welsh et al. 2012). The latter two systems were actually identified by eclipse timing. Even if the substellar component is not transiting the inner binary, it causes timing variations of eclipses of the binary system due to the finite velocity of light (light-time effect, hereafter LITE). The eclipses act as an accurate clock for detecting other objects revolving around the inner binary and to determine their orbital parameters from the observed calculated times of minima (O-C) in way similar to the solution of RV curves. The timing technique proved to be the most fruitful in detecting circumbinary planets.

The principal goal of the project DWARF is to detect circumbinary planets and/or substellar companions through the timing analysis of selected close eclipsing binaries (Pribulla et al. 2012).

\section{List of participating observatories}

SOAO, Korea; Terskol, Russia; OMU, Ankara, ITAP, Ege, Turkey; Kottamia, Egypt; MAO NASU, Lesniki, Ukraine; Rozhen, Bulgaria; Feleacu, Romania; Star Lesn, Kolonica, Roztoky, Slovakia; Patras, Greece; Astron. Station Vidojevica, Serbia; Szeged, Hungary; Torun̆, Poland; Brno, Prague, Czech Rep.; Hvar, Croatia; Graz, Austria; Catania, Italy; TLS, Jena, Kirchheim, Herges-Hallenberg, Trebur, Germany; LOAO, USA; $\dagger$

\section{References}

Broucke, R. A. 2001, Celestial Mechanics and Dynamical Astronomy, 81, 321

Doyle, R. L., Carter, J. A., Fabrycky, D. C., et al. 2011, Science, 333, 1602

Dvorak, R. 1984 (Celestial Mechanics), 34, 369

Konacki, M., Muterspaugh, M. W., Kulkami, S. R., \& Helminiak, K. G. 2009, ApJ, 704, 513

Lee, J. W., Kim, S. L., Kim, Ch. H., et al. 2009, AJ, 137, 3181

Muterspaugh, M. W., Konacki, M., Lane, B. F., \& Pfahl, E. 2007, in PLANETS IN BINARY STAR SYSTEMS, ed. N. Haghighipour (Springer).

Pierens, A. \& Nelson, R. P. 2008, A\& $A, 483,633$

Pribulla, T., Rucinksi, S. M., Blake, R. M., et al. 2009, AJ, 137, 3655

Pribulla T., et al. 2012, Astron. Nachr., 333, 754

Qian, S. B., Liu, L., Zhu, L. Y., et al. 2012, MNRAS, 422, 24

Welsh, W. F., Orosz, S. A., Carter, J. A., et al. 2012, Nature, 481, 475

$\dagger$ status as of April 22, 2012, not necessarily in that order 